\title{
UNA EXPERIENCIA DE EVALUACIÓN FORMATIVA EN EDUCACIÓN FÍSICA EN EDUCACIÓN SECUNDARIA
}

A experience formative assessment in Physical Education in Secondary Education

Experimente uma Avaliação formativa na Educação Física no Ensino Secundário

\section{Ivo Jiménez Barreto}

Universidad de La Laguna, España. Teléfono +34 676019088. Correo electrónico: ivoimenezbarreto@gmail.com

\begin{abstract}
Resumen
Se recoge una experiencia de innovación docente en la que se ha aplicado un sistema de evaluación formativa en el desarrollo de una situación de aprendizaje en la asignatura de Educación Física en la Etapa de Educación Secundaria. Para la valoración de la percepción del alumnado respecto a las ventajas e inconvenientes del sistema de evaluación, se administró un cuestionario a todo el alumnado al finalizar la situación de aprendizaje. Los resultados obtenidos muestran que el alumnado percibe como ventajas principales que fomenta la participación del alumnado en la evaluación de su aprendizaje, existe un mayor seguimiento y ayuda al alumnado por parte del profesorado, la evaluación atiende a un mayor número de aspectos y que se aprende más; y como inconvenientes más relevantes que exige una asistencia continua, tiene una dinámica poco conocida, y exige un mayor esfuerzo.
\end{abstract}

Palabras clave: Evaluación formativa; educación física; educación secundaria; metaevaluación

\begin{abstract}
It is an experience of teaching innovation in which a system of formative assessment has been applied in the development of a learning situation in the subject of Physical Education for the Secondary Education Stage. For the evaluation of the students' perception of the advantages and disadvantages of the evaluation system, a questionnaire was administered to all students at the end of the learning situation. The obtained results show that the students perceive as main advantages that encourages the participation of the students in the evaluation of their learning, there is a greater


monitoring and help to the students by the faculty, the evaluation attends to a greater number of aspects and that is learned plus; And as more relevant disadvantages that require continuous assistance, has a little known dynamics, and requires more effort.

Keywords: Formative assessment; physical education; Secondary education; Metaevaluation

\section{Resumo}

Uma experiência em inovação de ensino foi aplicado um sistema de avaliação formativa no desenvolvimento de uma situação de aprendizagem na disciplina de Educação Física para o Ensino estágio secundário é coletado. Para a avaliação da percepção dos alunos sobre as vantagens e desvantagens do sistema de avaliação, foi aplicado um questionário a todos os alunos no final da situação de aprendizagem. Os resultados mostram que os alunos percebidos como principais vantagens que incentiva a participação dos alunos na avaliação da aprendizagem, há um maior acompanhamento e apoio aos alunos pelos professores, a avaliação serve um número maior de questões e você aprende mais; e problemas mais relevantes que necessitam de assistência contínua, tem um pouco dinâmico conhecido, e requer mais esforço.

Palavras-chave: Avaliação formativa; Educação Física; Educação secundária; metaavaliação

\section{Introducción}

La evaluación formativa se sustenta en el seguimiento constante del proceso de enseñanza y aprendizaje, con el objetivo de mejorar dicho proceso (Scriven, 1967). Gracias a ello, el profesorado puede ajustar su propuesta metodológica a las necesidades formativas del alumnado, y recibir orientaciones precisas para regular su actividad académica (Brown \& Glasner, 2003). Para ello es necesario dotar a las actividades de evaluación de un carácter continuo, y aportar al alumnado un feedback formativo. En este caso, estaríamos hablando de evaluación para el aprendizaje (Álvarez, 2006), e incorporando cuestiones éticas al proceso de evaluación si abrimos vías de participación en la evaluación al alumnado. Desde este enfoque, evaluar supondría comprometerse a dotar a las actividades de evaluación de utilidad formativa para los agentes que 
participan en el proceso orientando el aprendizaje del alumnado más allá de acreditar el nivel alcanzado, y reorientando la enseñanza del profesorado. Ello implica concebir la educación como vía de emancipación que da acceso al saber (Álvarez, 2006).

\section{Contextualización}

La experiencia de innovación se desarrolló durante el curso 2014-15 en un centro público de Salamanca en la Etapa de Educación Secundaria (ESO). Se aplicó un sistema de evaluación formativa en el desarrollo de una situación de aprendizaje (bádminton) de 8 sesiones. Participaron 20 estudiantes de $4^{\circ}$ de ESO (4 alumnos y 16 alumnas), con edades comprendidas entre los 15 y 16 años.

Los objetivos de esta experiencia son: 1.- Identificar los rasgos caracterizadores de la evaluación formativa que contiene el sistema de evaluación aplicado; y 2.Conocer las ventajas y desventajas que el alumnado atribuye al sistema de evaluación formativa utilizado.

\section{Diseño y desarrollo}

Para dar respuesta al primer objetivo hemos tomado como referencia la propuesta de Navarro \& Jiménez (2012) en la que se propone y aplica un instrumento específico de metaevaluación didáctica para diagnosticar el carácter formativo de un determinado sistema de evaluación en Educación Física (Tabla 1).

Tabla 1.

Características del sistema de evaluación

\begin{tabular}{|c|c|c|c|c|c|c|c|c|c|c|c|}
\hline \multicolumn{12}{|c|}{ FICHA de METAEVALUACIÓN DIDÁCTICA } \\
\hline \multicolumn{3}{|c|}{ Profesor/a: I.J.B. } & \multicolumn{5}{|c|}{ Centro: I.E.S. Vía de la Plata } & \multicolumn{4}{|c|}{ U.D. Jugando al Bádminton } \\
\hline \multirow[t]{2}{*}{ Instrumentos } & \multicolumn{2}{|c|}{ Evaluación } & \multicolumn{2}{|c|}{ Implicación } & \multicolumn{3}{|c|}{$\begin{array}{c}\text { Momentos de la } \\
\text { evaluación }\end{array}$} & \multirow[t]{2}{*}{$\begin{array}{l}\text { Flujo de comunicación } \\
\text { o feedback formativo }\end{array}$} & \multicolumn{3}{|c|}{$\begin{array}{c}\text { Criterios de } \\
\text { evaluación }\end{array}$} \\
\hline & APR & ENS. & A & $\mathrm{P}$ & Ini. & Pro. & Fin. & & $\mathrm{C}$ & $\mathrm{M}$ & AF/S \\
\hline $\begin{array}{c}\text { Prueba de } \\
\text { preguntas } \\
\text { combinadas } \\
\end{array}$ & & & $\mathrm{X}$ & & $\mathrm{x}$ & & & $\begin{array}{l}\text { Verbal al grupo clase al } \\
\text { comienzo de la segunda } \\
\text { sesión }\end{array}$ & $\mathrm{X}$ & & \\
\hline $\begin{array}{l}\text { Lista de control } \\
1 \text { ámbito motor }\end{array}$ & $\mathrm{X}$ & & $\mathrm{X}$ & & $\mathrm{X}$ & & & $\begin{array}{l}\text { Verbal hacia todo el } \\
\text { grupo clase al comienzo } \\
\text { de la tercera sesión }\end{array}$ & & $\mathrm{X}$ & \\
\hline $\begin{array}{c}\text { Escala } \\
\text { descriptiva 1 }\end{array}$ & $\mathrm{X}$ & & $\mathrm{X}$ & & & $\mathrm{X}$ & & $\begin{array}{c}\text { Verbal pequeños grupos e } \\
\text { individuales }\end{array}$ & $\mathrm{X}$ & $\mathrm{X}$ & \\
\hline $\begin{array}{c}\text { Escala } \\
\text { descriptiva } 2\end{array}$ & $\mathrm{X}$ & & $\mathrm{X}$ & & & $\mathrm{X}$ & & $\begin{array}{c}\text { Verbal en pequeños } \\
\text { grupos al inicio de la } \\
\text { quinta sesión }\end{array}$ & & $\mathrm{X}$ & \\
\hline $\begin{array}{c}\text { Escala } \\
\text { descriptiva } 3\end{array}$ & $\mathrm{X}$ & & $\mathrm{X}$ & & & $\mathrm{X}$ & & $\begin{array}{c}\text { Verbal en pequeños } \\
\text { grupos al inicio de la } \\
\text { quinta sesión } \\
\end{array}$ & & $\mathrm{X}$ & $\mathbf{x}$ \\
\hline Lista de control 2 & $\mathrm{X}$ & & $\mathrm{X}$ & $\mathrm{X}$ & & $\mathrm{X}$ & & $\begin{array}{l}\text { Verbal de manera } \\
\text { individual }\end{array}$ & $\mathrm{X}$ & $\mathrm{X}$ & $\mathbf{x}$ \\
\hline Diario de clase & & $\mathbf{X}$ & & $\mathbf{x}$ & & $\mathbf{X}$ & & & $\mathbf{x}$ & $\mathbf{X}$ & $\mathbf{x}$ \\
\hline
\end{tabular}

Nota: APR.= Aprendizaje; ENS.= Enseñanza; A=Alumnos; P=Profesor; Ini.=Inicial; Pro.=Proceso; Fin.=Final; C=Ámbito cognitivo; $\mathrm{M}=$ Ámbito motor; $\mathrm{A} / \mathrm{F}=$ Ámbito afectivo-social 
Para conocer la percepción del alumnado respecto a las ventajas e inconvenientes del sistema de evaluación aplicado, se administró un cuestionario al finalizar la situación de aprendizaje. El cuestionario empleado es una adaptación al ámbito de la Educación Secundaria de los ítems de las preguntas 12 y 13 de la versión del cuestionario empleado en el curso 13-14 en la Red Nacional de Evaluación Formativa y Compartida (Castejón, Santos \& Palacios, 2015). Las adaptaciones realizadas fueron validadas por dos expertos, profesores universitarios con una amplia experiencia investigadora. Además la escala resulta ser un instrumento válido para ayudar al profesorado a analizar las percepciones de los estudiantes en relación con la evaluación formativa (Castejón, Santos \& Palacios, 2015).

Los datos del cuestionario fueron sometidos a un análisis descriptivo, para lo que se empleó el programa SPSS v.21. Los resultados se presentan diferenciando las ventajas e inconvenientes de las tres dimensiones consideradas: aprendizaje, compromiso y proceso (Jiménez, Navarro, Pintor, \& Souto, 2013).

\section{Evaluación y conclusiones}

El empleo de la ficha de metaevaluación didáctica ha permitido confirmar que el sistema de evaluación desarrollado cumple con los rasgos caracterizadores de una evaluación formativa, y advertir de la relación entre el excesivo número de instrumentos de evaluación movilizados y el aumento de la complejidad, lo que puede poner en riesgo la viabilidad del sistema de evaluación.

En cuanto a las ventajas del sistema de evaluación empleado, en la dimensión ‘aprendizaje’ se destacan que fomenta la participación de todo el alumnado, se percibe un mayor aprendizaje y se evalúan diversos aspectos. En la dimensión ‘compromiso’ se valora la existencia de una negociación previa y la demanda de mayor responsabilidad. En la dimensión 'proceso' existe un mejor seguimiento, se valora el trabajo diario, y que el feedback recibido permite la mejora del aprendizaje. En cuanto a los inconvenientes, se destaca en la dimensión 'compromiso' que exige una asistencia continua y una participación activa, y en la dimensión proceso la falta de hábito con la dinámica de evaluación seguida.

Como aspecto a mejorar es necesario modular la complejidad del modelo de evaluación con alumnado que no esté familiarizado con la dinámica de trabajo de este sistema de evaluación. 


\section{Referencias}

Álvarez, J. M. (2006). La evaluación educativa al servicio de quien aprende: el compromiso necesario con la acción crítica. En V. López., R. Monjas., y C. Vallés (Eds.): Actas I Congreso Nacional de Evaluación Formativa en Docencia Universitaria. Segovia: Universidad de Valladolid.

Brown, S., \& Glasner, A. (Eds.) (2003). Evaluar en la Universidad. Problemas y nuevos enfoques. Madrid: Narcea.

Castejón, F. J., Santos, M. L., \& Palacios, A. (2015) Cuestionario sobre metodología y evaluación en formación inicial en educación física. Revista Internacional de Medicina y Ciencias de la Actividad Física y el Deporte, vol. 15(58), 245-267. Recuperado de http://cdeporte.rediris.es/revista/revista58/artescala566.htm

Jiménez, F., Navarro. V., Pintor, P., \& Souto, R. (2013). Percepción del alumnado de las dimensiones 'proceso', 'aprendizaje’ y 'compromiso' en el marco de los sistemas de evaluación formativa. En Ma J. Cuéllar Moreno., y J. O’Dwyear Acosta (Eds). Innovación en las enseñanzas universitarias: experiencias presentadas en las III Jornadas de Innovación Educativa de la ULL, 145-160. San Cristobal de La Laguna: Servicio de Publicaciones de la Universidad de La Laguna.

Navarro, V., \& Jiménez, F. (2012). La mejora en la evaluación formativa de maestros de educación física a través de un instrumento de metaevaluación didáctica. Revista Internacional de Ciencias del Deporte, 27, 63-79.

Scriven, M. (1967). The metodology of evaluation, In R. E. Stake, Perspectives of curriculo evaluation. (vol. 1, pp. 39-55). Chicago, IL: Rand McNally. 